\title{
Optimal Measurement Placement Using PSO for State Estimation
}

\author{
Hazwani Mohd Rosli \\ Department of Electrical \\ Engineering \\ University of Malaya \\ Kuala Lumpur, Malaysia \\ wanirosli@siswa.um.edu.my
}

\author{
Hazlie Mokhlis \\ UMPEDAC, Faculty of Engineering \\ University of Malaya \\ Kuala Lumpur, Malaysia \\ hazli@um.edu.my
}

\author{
Ab. Halim Abu Bakar \\ UMPEDAC, Faculty of Engineering \\ University of Malaya \\ Kuala Lumpur, Malaysia \\ a.halim@um.edu.my
}

\begin{abstract}
This paper presents an effective method based on Particle Swarm Optimization (PSO) to identify the optimal measurement placement of power system state estimation. The main objective is to simplify the complexity in finding the best measurement placement and provide a high accuracy level of estimated state. The effectiveness of the proposed method is tested using the IEEE 30 bus system. Pseudo measurements of load injection are included as measurement data in assisting the state estimation computation.
\end{abstract}

Keywords - Measurement placement; Particle Swarm Optimization; state estimation and observability.

\section{INTRODUCTION}

State estimation is an essential tool in monitoring the operating conditions of the power system. It improves system security, data accuracy and reduce the measurement and telemetry cost. State estimator generates system states of voltage magnitude and phase angles based on available measurement data and network configurations. Network parameters and topology basically are perfectly known. On the other hand, line power flows, bus power injections, bus voltage magnitudes and line current magnitudes are necessarily be measured at a particular location. Basically, not all measurement data contribute in producing the best state estimation for optimal monitoring of power system. Some measurements may contain bad data which affect the state estimator performance. The result of state estimation depends on the location and types of measurements. With suitable placement of meters in all buses and lines, state estimation will have better performance.

Observable network condition with sufficient available measurement data is also necessary for state estimation to execute. The observability of a power system depends on the number of measurement data as well as the topology of the network. Increasing number of measurement data improves observability. However, it is not possible to place meters at all nodes and lines due to high investment cost. Therefore, much research has been conducted on observability analysis and optimal measurement placement of the network such in [1-4]. If the network is observable, state estimation may proceed; otherwise, pseudo-measurements must be added to the measurement set to allow an estimate of the state vector to be computed [3, 4]. Pseudo measurements are typically bus injections generated from load forecasts or historical data. Since these are not actually measured quantity, the accuracy is however much less compared to the real-time measurements.

Several methods have been proposed by researchers on measurement placement technique for power system state estimation [4-6]. In [7], Abur and Gou uses the idea of numerical observability. Simultaneous placement of a minimal set of pseudo measurements makes the system observable. The analysis from [7] has suggested of employing the minimum condition number of the measurement Jacobian matrix. Singular Value Decomposition approach has been used to solve the state estimation. Celik and Liu [6] proposed an incremental measurement placement algorithm in order to increase local redundancy and strengthen network observability and thereby enhance the quality of state estimation solutions.

Although there are various methods for optimal measurement placement, the computational time still is a burden due to the complex algorithm. As in [7] which applied the basis of sequential meter addition-elimination process also has the limitation of not being suitable for too many measurements due to excessive computation time. The method given in [5] presents a repetitive procedure of temporarily eliminates each possible location one at a time until the condition number of the corresponding matrix is satisfied in which results in relatively large time of calculations. Therefore, this paper proposes an optimization method of PSO to assist in locating the optimal measurement placement for power system state estimation. The proposed algorithm randomly searches the best location of measurement placement based on the minimum error of state estimation. The main concern is to achieve a high accuracy level for state variables from the optimal measurement placement obtained. In comparison to traditional methods, PSO able to search the optimal measurement placement without having to test possible location one after another since PSO is an optimization method. It search better position of measurement placement by iteratively improve the random position guided by the selected constraint and objective function. Pseudo measurements are used to avoid the singularity in gain matrix and recover the observability of the system.

This paper first presents an overview of state estimation formulation in Section II. The proposed method of PSO to 
locate the optimal measurement placement of a network system is then described in Section III. The results of the estimation accuracy obtained from the optimal meter location and the consistency of the method are discussed in Section IV. Comparison is made to several numbers of measurement placement to find the optimal measurement placement which produces the best state estimation.

\section{STATE ESTIMATION FORMULATION}

A typical algorithm for state estimation uses the Weighted Least Squares (WLS) method that minimizes the sum of the squared weighted errors between estimated and actual measurements. The nonlinear relationship between measurements and state variables is shown as follows:

$$
z=h(x)+v
$$

where

$\begin{array}{ll}z & \text { measurement vector } \\ x & \text { state vector } \\ h(x) & \text { non-linear measurement function } \\ v & \text { measurement noise vector }\end{array}$

Based on WLS method, the state vector is determined in minimizing the following function:

$$
J(x)=\sum_{i=1}^{m}\left(z_{i}-h_{i}(x)\right)^{2} / R_{i i}
$$

where $R_{i i}$ is a diagonal matrix called the noise covariance matrix. During the state estimation process, the power system network is defined to be observable if the gain matrix of Eq. (3) is non singular.

$$
G\left(x^{k}\right)=H^{T} R^{-1} H
$$

Another significant indication of the performance of the state estimation is the variant of the state estimation error. It can be shown that the state estimation error defined as

$$
\alpha=x^{t}-x^{e}
$$

which has the covariance matrix of

$$
\begin{gathered}
C=E\left[\left(x^{t}-x^{e}\right)\left(x^{t}-x^{e}\right)^{T}\right] \\
=\left(H^{T} R^{-1} H\right)^{-1}
\end{gathered}
$$

where $E$ stands for expectation, $x^{t}$ is the true state and $x^{e}$ is the estimated state. Statistically, the state estimation error variances represent the possible "distance" of the estimates from their true values. Small variances provide better solution for state estimation. Hence, the minimization of Eq. (5) is used as an objective function to identify the optimal measurement placement of the system in this proposed method.

The network system is said to be observable if and only if the observability matrix, $H$ is full rank. Thus, the algorithm is constrained by full rank of matrix $H$ to have a well topology network of the system. In addition, to have the possibility of estimation of the state vector, there should be at least $2 n-1$ measurements where $n$ is the number of buses. Therefore, pseudo measurement is necessary as measurement data to compute the state estimation in this procedure since line flow measurement data have not taken into account.

\section{PROPOSED PARTICLE SWARM OPTIMIZATION (PSO) ALGORITHM FOR OPTIMAL PlaCEMENT}

Particle Swarm Optimization is one of the heuristic methods used by researchers to solve many problems related to power systems. The basic idea of the PSO is based on the social behavior (foraging) of organisms such as fish (schooling) and bird (flocking). The birds or fish will move to the food in certain speed or position. Their movement will depend on their own experience and experience from other "friends" in the group ( $P_{b e s t}$ and $\left.G_{b e s t}\right)$. The new velocity, $V_{j}^{k+1}$ and the new position, $X_{j}^{k+1}$ for the fish or birds are obtained using Eq. (6) and (7).

$$
\begin{gathered}
V_{j}^{k+1}=\omega \times V_{j}^{k}+C_{1} \times \operatorname{rand}_{1} \times\left(P_{\text {best }_{j}}^{k}-X_{j}^{k}\right) \\
+C_{2} \times \operatorname{rand}_{2} \times\left(G_{\text {best }_{j}}^{k}-X_{j}^{k}\right) \\
X_{j}^{k+1}=X_{j}^{k}+V_{j}^{k+1}
\end{gathered}
$$

where $V_{j}^{k}$ is the velocity of particle $j$ in the iteration $k, X_{j}^{k}$ is the position of particle $j$ in the iteration $k, \operatorname{rand}_{1}$ and rand $_{2}$ are the random numbers between 0 and $1 . P_{\text {best }_{j}}^{k}$ is the best value of the fitness function that has been achieved so far by any particle. Constant $C_{1}$ and $C_{2}$ are acceleration coefficients of the random acceleration terms which are usually in the range of 1.0 to 2.0 . While low values allow particles to move away from the target region before they are pulled back, higher values result in sharp movements toward the target region. The inertia weight $\omega$ is typically set according to the following equation:

$$
\omega(t)=\omega_{\max }-\frac{\omega_{\max }-\omega_{\min }}{t_{\max }} \times t
$$

In Eq. (8), $t_{\text {max }}$ is the maximum number of iterations and $t$ is the current iteration number. $\omega_{\max }$ and $\omega_{\min }$ are maximum and minimum of the inertia weights respectively. The process of implementation of PSO algorithm is as follows: 
Step 1: Input slack bus voltage, real and pseudo measurement data, bus limit, and upper limit of objective function.

Step 2: Initialize the PSO parameters. Set up the set of parameters of PSO such as, number of particles $N$, acceleration coefficients, $C_{1}$ and $C_{2}$ and maximum iteration number.

Step 3: Calculate the state estimation using weighted least squares method.

Step 4: Randomly generates an initial population (array) of particles with random positions and velocities on dimension in the solution space. Set the iteration counter $i=0$.

Step 5: For each particle if the bus number is within the limits, calculate the state estimation using weighted least squares method. Otherwise, that particle is infeasible.

Step 6: Record and update the best values. The two best values are recorded in the searching process. Each particle keeps track of its coordinate in the solution space that is associated with the best solution it has reached so far. This value is recorded as $P_{b e s t}$. Another best value to be recorded is $G_{\text {best }}$, which the overall best value is obtained so far by any particle. $P_{\text {best }}$ and $G_{\text {best }}$ are the minimum value of the objective function. This step also updates $P_{\text {best }}$ and $G_{\text {best }}$. At first, we compare the fitness of each particle with its $P_{b e s t}$. If the current solution is better than its $P_{\text {best }}$, then replace $P_{\text {best }}$ by the current solution. Then, the fitness of any particle is compared with $G_{\text {best }}$. If the fitness of any particle is better than $G_{\text {best }}$, then replace $G_{\text {best }}$.

Step 7: Update velocity and position of the particles. Eq. (6) is applied to update the velocity and position of the particles. The velocity of a particle represents a movement of the selected bus. Meanwhile, Eq. (7) is applied to update the position of the particles.

Step 8: End conditions. Check the end condition, if it is reached the algorithm, stop, otherwise repeat step 3-7 until the end conditions are satisfied.

In this work, the particles are the measurement meter $(\mathrm{M})$ as shown in Eq. (9) below:

$$
X_{\text {particle }}=\left[M_{1}, M_{2}, \ldots ., M_{\alpha}\right]
$$

where $\alpha$ is the location of measurement placement.

\section{Simulation And AnAlysis Of The Results}

In this section, the practical results associated with PSO method applied to state estimation for optimal measurement placement are obtained. For this purpose, the IEEE 30 bus system in Fig. 1 is used as the test network while real measurement data is taken from the load flow program using Newton Raphson method.

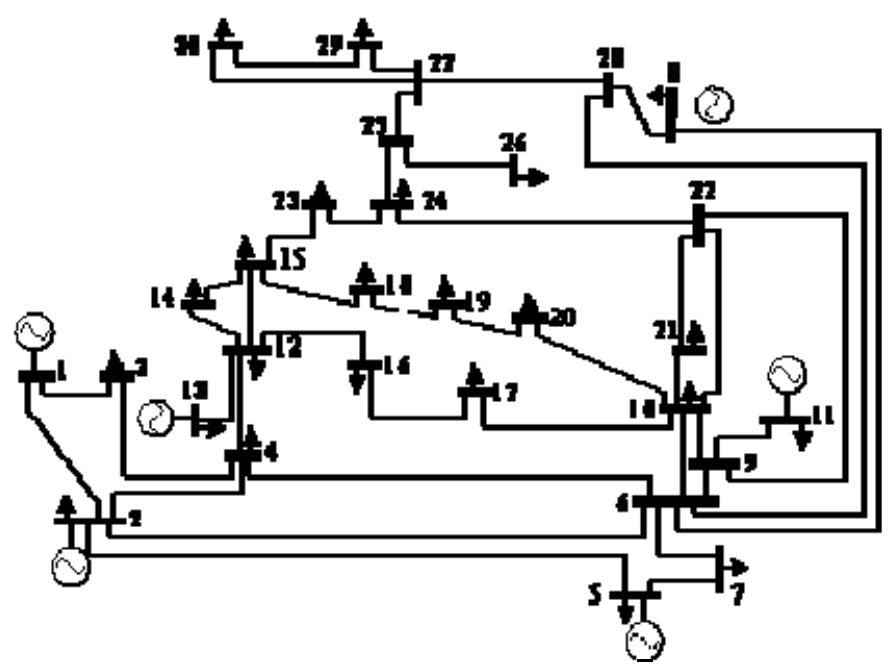

Figure 1. IEEE 30 bus system network

In the test, three different errors of pseudo measurement data are considered i.e. $20 \%, 30 \%$, and $40 \%$ measurement errors. The location of the meter is determined using the proposed method for each different error as well as the different number of measurement meter placement. A slack bus voltage and bus power injections are used as measurement data throughout the computation. The analysis is divided into several cases for further discussion.

\section{A. Results of Using Pseudo Measurement}

Initially the simulation is run to compare the result of estimated voltage by using pseudo measurement and real measurement data of all power injection on the network. The measurement meter is assumed to be on the bus network by using real measurement data. Therefore, Fig. 2 shows the estimated voltage by using $20 \%$ error of pseudo measurement data and $1 \%$ error of real measurement data which indicate that each bus of the IEEE 30 bus network has measurement meter placement.

The pattern of the estimated state curve of using pseudo measurement data has less accuracy comparable to curve of using real measurement data. However, if only a certain number of real measurement data without pseudo measurement is used to compute the state estimation, the result gives undefined value. Hence, it shows that pseudo measurement is necessary in assisting to solve the state estimation. 


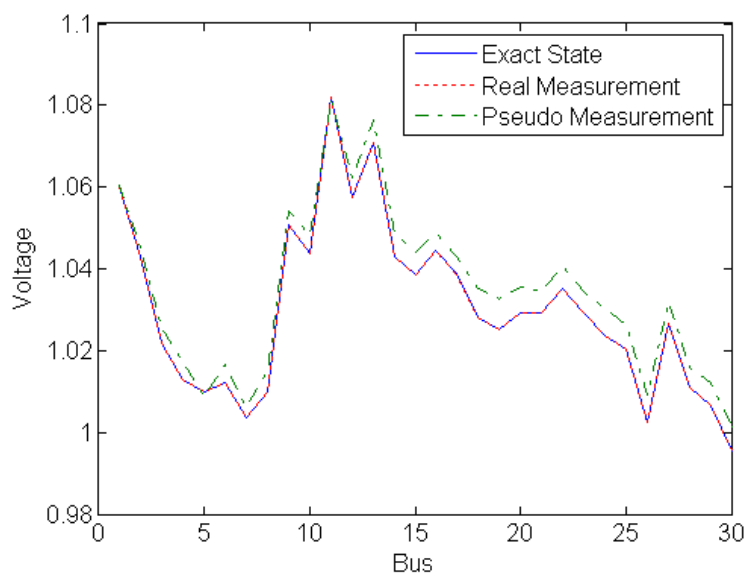

Figure 2. Estimated state for IEEE 30 bus test system using $20 \%$ error of pseudo measurement and $1 \%$ error of real measurement data

The algorithm then computes with $30 \%$ and $40 \%$ error of pseudo measurement data. The result in Fig. 3 shows that the estimated states curve with higher measurement error is far from the exact state.

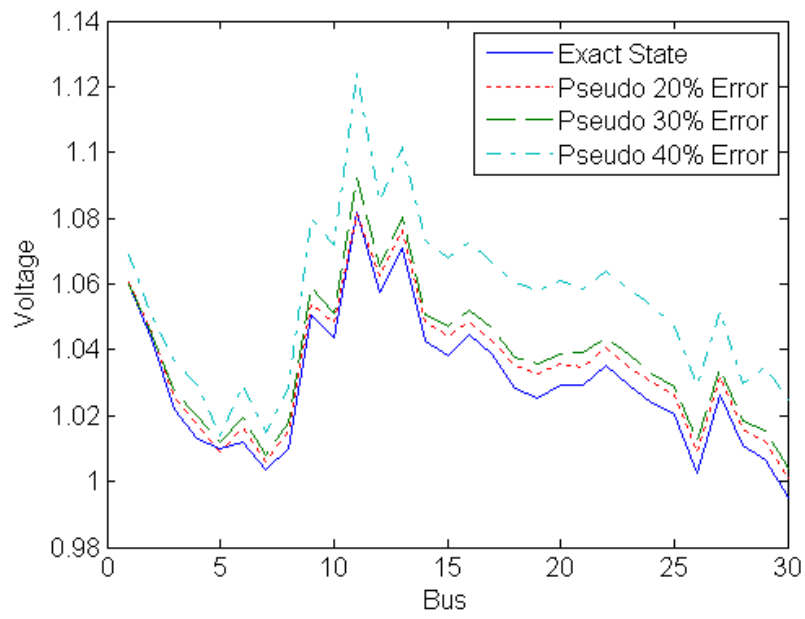

Figure 3. Estimated state for IEEE 30 bus test system using pseudo measurement data with different measurement error

Then, the accuracy of state estimation is calculated using Eq. 5 to study the effect of different accuracy of pseudo measurement on the performance of state estimator. The result of state estimation accuracy is shown in Table 1.

TABLE I. STATE COVARIANCE ERROR OF DIFFERENT TYPES AND MEASUREMENT ERROR

\begin{tabular}{|c|c|c|}
\hline $\begin{array}{c}\text { Types of } \\
\text { Measurement }\end{array}$ & Measurement Error & $\begin{array}{c}\text { State Covariance } \\
\text { Error }\end{array}$ \\
\hline Real & $1 \%$ & 0.0382 \\
\hline Pseudo & $20 \%$ & 0.1699 \\
\hline Pseudo & $30 \%$ & 0.1978 \\
\hline Pseudo & $40 \%$ & 0.2227 \\
\hline
\end{tabular}

It can be shown that the accuracy of state estimation is affected by different types and error of measurement data. The state estimation with real measurement has better accuracy compare of using pseudo measurement data. Meanwhile, the error of state estimation increase with increasing of pseudo measurement error. Therefore, it shows that the accuracy of the state estimation decreases due to the error of measurement data.

\section{B. Results of Using Optimal Measurement Placement}

The proposed algorithm of searching the optimal measurement placement for state estimation is then computed to identify the optimal location of meter placement on the network. Initially, total number of three optimal meter measurement is used for the analysis. The algorithm is tested with different error on pseudo measurement data as in Table 2.

Table 2 shows the best result of optimal measurement placement and the minimum objective function for each pseudo measurement error. The results shown verify that the optimal location of measurement placement is similar for different error of pseudo measurement data. The minimum state covariance error value is different due to different pseudo measurement error.

TABLE II. STATE COVARIANCE ERROR FOR DIFFERENT ERROR OF PSEUDO MEASUREMENT AND THE OPTIMAL PLACEMENT

\begin{tabular}{|c|c|c|}
\hline $\begin{array}{c}\text { Pseudo } \\
\text { Measurement } \\
\text { Error }\end{array}$ & $\begin{array}{c}\text { Minimum State } \\
\text { Covariance Error }\end{array}$ & $\begin{array}{c}\text { Optimal Placement } \\
\text { Bus }\end{array}$ \\
\hline $20 \%$ & 0.1196 & $1,2,5$ \\
\hline $30 \%$ & 0.1375 & $1,2,5$ \\
\hline $40 \%$ & 0.1502 & $1,2,5$ \\
\hline
\end{tabular}

In order to prove that the location of the meter obtained by PSO method is an optimal measurement placement, the results of the estimated state are compared with the exact state as in Fig. 4, 5 and 6 for $20 \%, 30 \%$ and $40 \%$ pseudo measurement error respectively. Hence, it can be shown in Fig. 4, 5 and 6 that the measurement data with optimal meter placement reduces the error of estimated state compared to the measurement by using only pseudo measurement data.

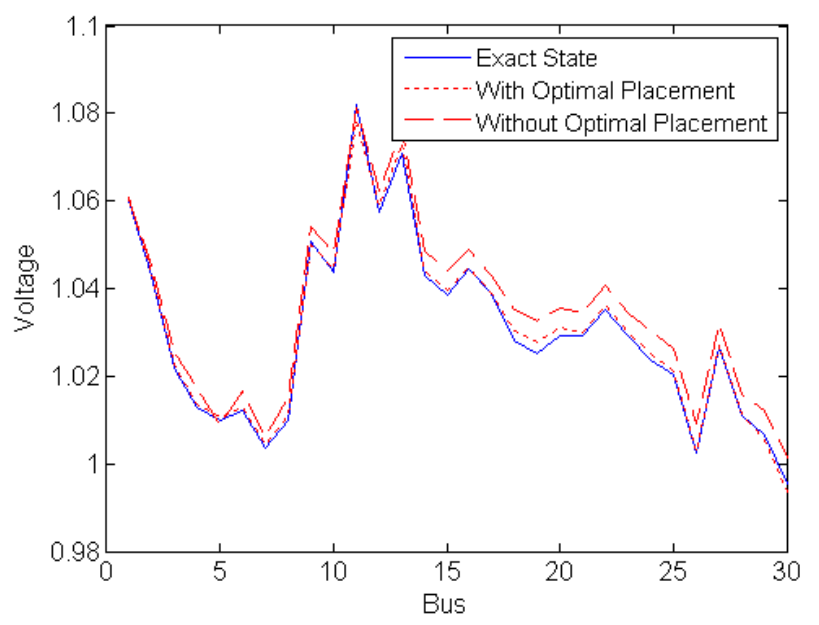

Figure 4. Estimated State of $20 \%$ Pseudo Measurement Error with Optimal Measurement Placement 


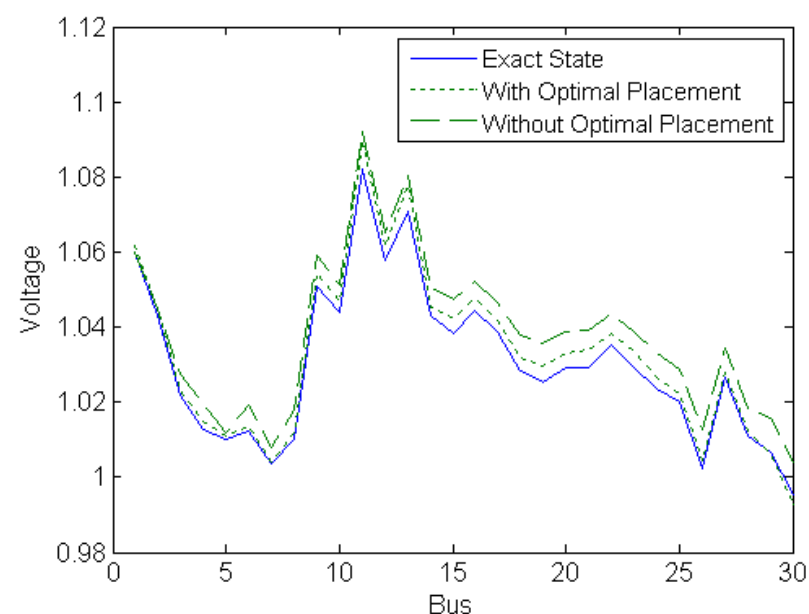

Figure 5. Estimated State of 30\% Pseudo Measurement Error with Optimal Measurement Placement

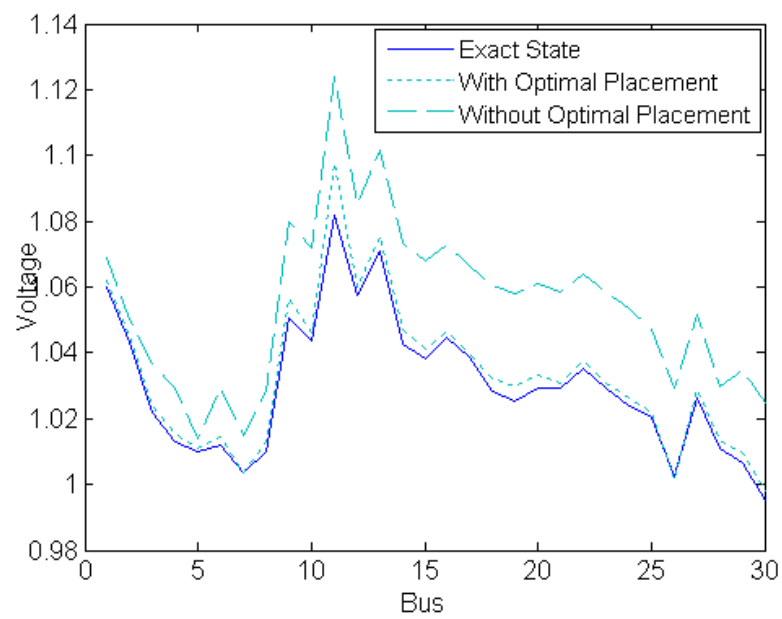

Figure 6. Estimated State of $40 \%$ Pseudo Measurement Error with Optimal Measurement Placement

Subsequently, the state covariance error is calculated as in Table 3. It shows that the state estimation with optimal measurement placement for all different error of data measurement have much better accuracy compare to the estimated state without optimal measurement placement. By varying the error of pseudo measurement, it can be concluded that the computation of state estimation using the selected optimal measurement placement can achieve high accuracy estimation even for all different measurement error.

TABLE III. STATE COVARIANCE ERROR OF USING OPTIMAL MEASUREMENT PLACEMENT

\begin{tabular}{|c|c|c|}
\hline \multirow{2}{*}{$\begin{array}{c}\text { Pseudo } \\
\text { Eeasurement } \\
\text { Error }\end{array}$} & \multicolumn{2}{|c|}{ State Covariance Error } \\
\cline { 2 - 3 } & $\begin{array}{c}\text { Without Optimal } \\
\text { Measurement } \\
\text { Placement }\end{array}$ & $\begin{array}{c}\text { With Optimal } \\
\text { Measurement } \\
\text { Placement }\end{array}$ \\
\hline $20 \%$ & 0.1699 & 0.1196 \\
\hline $30 \%$ & 0.1978 & 0.1375 \\
\hline $40 \%$ & 0.2227 & 0.1502 \\
\hline
\end{tabular}

\section{Results with Different Number of Measurement Placement}

After the optimal measurement for three locations is identified, the simulation is then proceed for different number of measurement placement. Example of five different numbers of measurement placement is used for the analysis. Table 4 shows the location for optimal measurement placement obtained by the PSO method with different number of measurement placement.

TABLE IV. LOCATION OF OPTIMAL PLACEMENT FOR DIFFERENT NUMBER OF MEASUREMENT PLACEMENT

\begin{tabular}{|c|l|c|}
\hline No. of Meter & \multicolumn{1}{|c|}{$\begin{array}{c}\text { Optimal Meter } \\
\text { Placement Bus }\end{array}$} & $\begin{array}{c}\text { State } \\
\text { Covariance } \\
\text { Error }\end{array}$ \\
\hline 3 & $1,2,5$ & 0.1196 \\
\hline 4 & $1,2,5,30$ & 0.1132 \\
\hline 5 & $1,2,5,7,30$ & 0.1074 \\
\hline 6 & $1,2,5,7,10,30$ & 0.1038 \\
\hline 7 & $1,2,5,7,10,11,30$ & 0.0955 \\
\hline
\end{tabular}

It can be shown that as the number of optimal measurement placement increase, the state covariance error decrease. Hence, the accuracy of state estimation can be increased by adding more number of measurement placements.

\section{Consistency of the PSO method}

The algorithm of the proposed method is executed for 50 times and 300 iterations of each time by using three optimal measurement placement to study the consistency of the method. Graph plot in Fig. 7 shows that the objective function reaches the minimum value inconsistently for each run of the program. It shows that the method produces inconsistent results for measurement placement of state estimation.

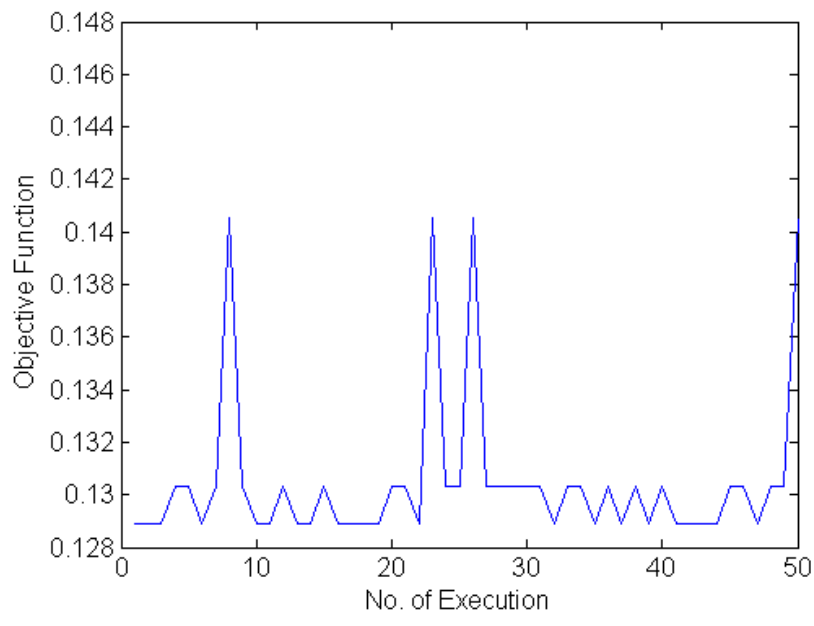

Figure 7. Objective function for 50 times of execution 
The pattern of objective function for 300 iterations of each execution is plotted as in Fig. 8. It can be seen there are a certain number of objective functions that does not reach the minimum value. This may due to the small number of population or maximum iteration. Therefore, the program has to be executed for several times in order to get the optimal measurement placement with the best quality of state estimation.

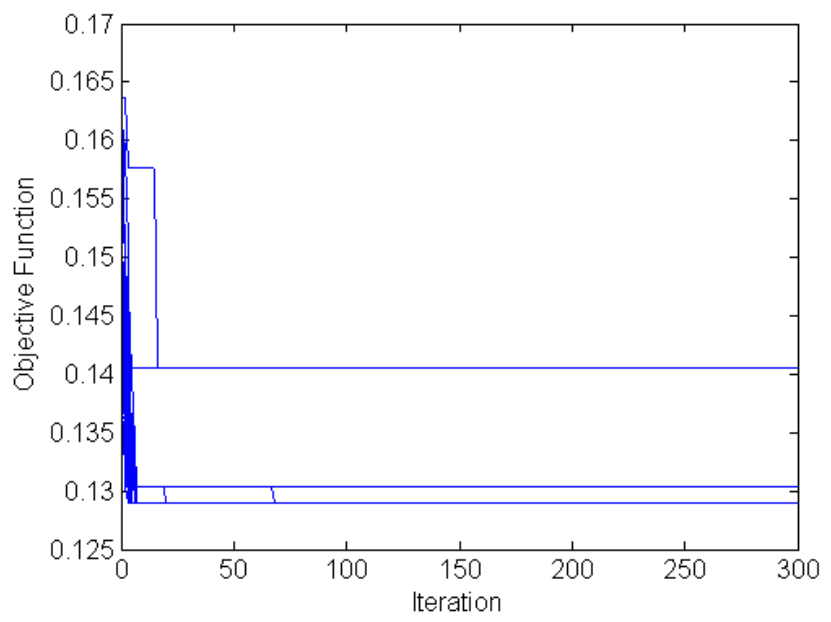

Figure 8. Objective function for 300 iteration of each execution

\section{CONCLUSION}

In this paper, an optimal measurement placement for state estimation by using PSO method is presented. The main objective of this proposed method is to randomly search the best possible measurement placement of the system which produces the best quality of the state variable. According to the conventional method of finding the optimal measurement placement, the system state variable is obtained by sequential addition-elimination process in which consume relatively large computation time. The complexity of the algorithm is effectively reduced by using the optimal method of PSO which use randomization techniques of selecting the best possible location of measurement placement.

It is observed that the proposed scheme manages to obtain the optimal measurement placement for state estimation in constraint of the state estimation objective function. By adding the redundancy of pseudo measurement, the computation of state estimation is assisted in terms of avoiding the singularity of gain matrix. The simulation results show that the accuracy of estimated state is increasing as number of measurement placements is added. Hence, it can be concluded that the best quality of state estimation is achievable by using only certain number of meters at the optimal measurement placement. However, the inconsistency of PSO method may affect the quality of the program computation. Therefore, the original PSO will be improved in future to achieve better consistency in producing the result.

\section{ACKNOWLEDGEMENT}

This work was supported by the Malaysian Government and University of Malaya, Kuala Lumpur under HIR/MOHE research grant (Grant Code: D000004-16001) and postgraduate research fund, IPPP (Grant Code: PS009-2012A).

\section{REFERENCES}

[1] A. Monticelli, F.F.Wu, "Network observability: theory," IEEE Trans. on Power App. \& Syst., May 1985. Vol. PAS-104, No. 5, pp. 1042-1048.

[2] A. Monticelli, F.F.Wu, "Network observability: Identification of Observable Islands and Measurement Placement," IEEE Trans. Power Syst., May 1985. Vol. PAS-104, No. 5, pp. 1035-1039.

[3] K. A. Clements, G.R. Krumpholz., P. W. Davis, "Power System State Estimation with Measurement Deficiency: An Observability/Measurement Placement Algorithm," IEEE Trans. on Power App. \& Syst., July 1983. Vol. PAS-102, No.7, pp. 2012-2020.

[4] Bei Gou, A. Abur, “An Improved Measurement Placement Algorithm for Network Observability,” IEEE Trans. on Power Syst., Nov. 2001. Vol. 16, No. 4, pp. 819-824.

[5] C. Madtharad, S.Premrudeepreechacharm, "Measurement Placement Method for Power System State Estimation: Part I.," 2003, IEEE Power Engineering Society General Meeting. pp. 1629-1632.

[6] M. K. celik, W.-H.E.Liu, “An Incremental Measurement Placement Algorithm for State Estimation," IEEE Trans. on Power Syst., Aug. 1995. Vol. 10, No. 3, pp. 1698-1702.

[7] Park Y. M., Moon Y.H., Choo J. B., Kwon T. W, "Design of reliable measurement system for state estimation, " IEEE Trans. on Power Syst., Aug. 1988. Vol. 3, No. 3, pp. 830-836.

[8] J. Kennedy, R.Eberhart, "Particle Swarm Optimization. in Proc. IEEE Int. Conf. Neural Networks," 1995. Perth, Australia.

[9] Y. Fukuyama, e.al, "A particle swarm optimization for reactive power and voltage control considering voltage security assesment," IEEE Trans. Power Syst., Nov. 2000. vol. 15, pp. 1232-1239. 